\title{
Glycosphingolipids: structure and function
}

\author{
Charles C. Sweeley \\ Department of Biochemistry, Michigan State University, East \\ Lansing, Michigan 48824 U.S.A.
}

\begin{abstract}
Glycosphingolipids occur in most higher organisms as minor constituents of the plasma membranes of cells. More than 200 compounds in this class have been isolated and chemically characterized. In this paper, we compare the structures of several complex glycosphingolipids and discuss the utility of fast atom bombardment mass spectrometry in the MS/MS mode with collision-assisted dissociation of the ceramide ion to study details of the ceramide structure. The function of several gangliosides in the regulation of growth of cultured cells is also discussed.
\end{abstract}

\section{INTRODUCTION}

Glycosphingolipids are amphipathic membrane-bound constituents of most plants, animals and marine organisms. This interesting and important class of natural products was first described in the 19th century by Johann L.W. Thudichum, a student of von Leibig, who discovered "cerebroside" (Fig. 1) in extracts of human brain. He also reported that cerebroside contained a unique long-chain base constituent ("sphingosine"), which could be liberated by barium hydroxide hydrolysis of the lipid. The complete structures of sphingosine and cerebroside were not completely elucidated until 1947 (1). A major stimulus to study glycosphingolipid chemistry and biochemistry was the discovery of an abnormal lipid called "substanz $X "$ in brain tissue from an infant with amaurotic idiocy (Tay-Sachs disease) by Ernst Klenk in 1935 (2). Klenk later isolated "neuraminic acid" from a methanolysate of "Substanz $X_{-}$" partially defining the first of a large group of substances known as gangliosides. The Tay-Sachs ganglioside structure (Fig. 1) was firmly established in 1963 by Kuhn and Wiegandt (3).

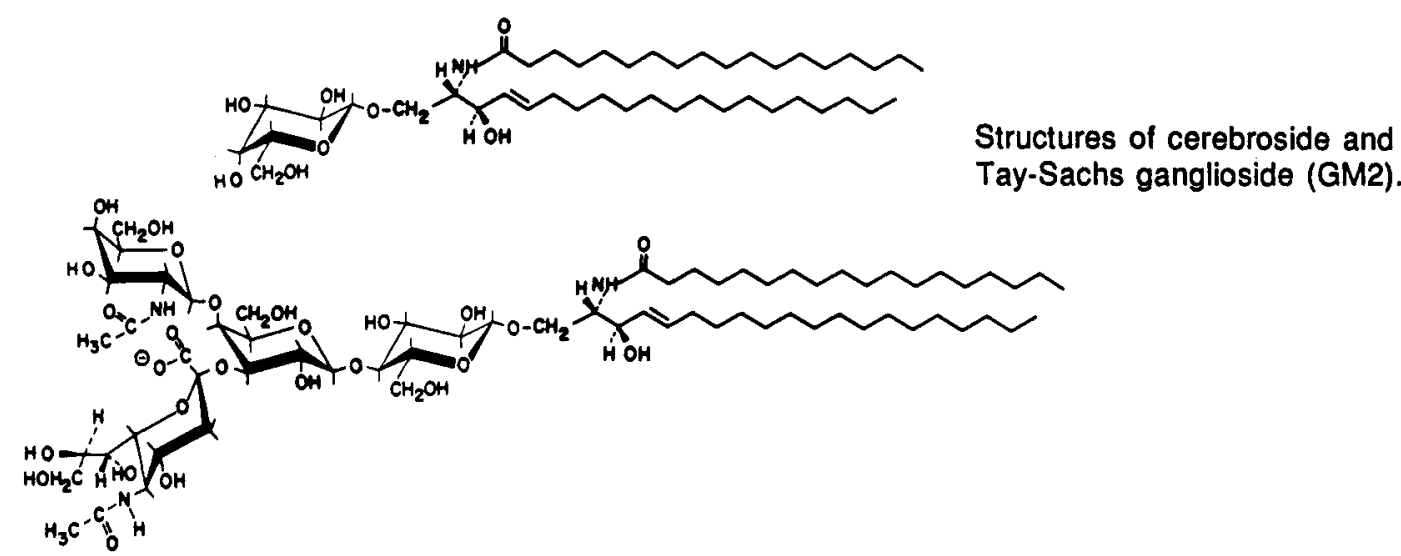

This paper is devoted to a brief examination of the chemical structures of several recently characterized glycosphingolipids, the application of fast-atom-bombardment mass spectrometry to structural studies, and the evolving role of gangliosides on the surface of mammalian cells in the regulation of cell growth.

\section{STRUCTURE}

The hydrophobic lipid moiety of glycosphingolipids that is anchored in the plasma membrane of cells is called ceramide. It is composed of a long-chain aliphatic aminoalcohol called a sphingoid base, of which there are several kinds, and a fatty acid that is covalently attached in an amide linkage to the sphingoid base. The most common constituents of ceramide are listed in Table I. The ceramide fraction of most glycosphingolipids is a mixture of molecular species of one or more of the sphingoid bases and several of the fatty acids. 
Table I Constituents of Ceramide

Sphinooid Base

\begin{tabular}{|c|c|}
\hline $\begin{array}{l}\text { Sphinganine }{ }^{1} \quad(d 18: 0)^{2} \\
\text { rans- } \Delta^{4}-\text { Sphingenine }^{3} \quad(d 18: 1) \\
\text { 4-D-Hydroxysphinganine (t18:0) }\end{array}$ & 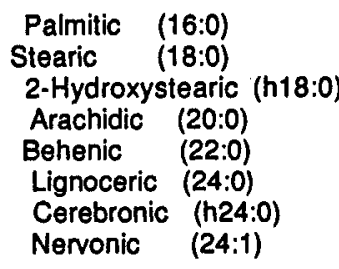 \\
\hline \multicolumn{2}{|c|}{$\begin{array}{l}{ }^{1} \text { The sphingoid base nomenclature is based on } \\
\text { (2S,3R)-2-amino-1,3-octadecanediol, called } \\
\text { sphinganine (4). }\end{array}$} \\
\hline \multicolumn{2}{|c|}{$\begin{array}{l}{ }^{2} \text { Abbreviations of sphingoid bases denote the number } \\
\text { of carbon atoms:double bonds and hydroxyl consti- } \\
\text { tuents ( } d \text { or } t \text { ). Fatty acids are abbreviated as chain } \\
\text { length:double bonds and presence or absence of } \\
\text { hydroxyl }(h) \text { groups on } \mathrm{C}-2 \text {. }\end{array}$} \\
\hline 90 & \\
\hline
\end{tabular}

The carbohydrate chain of glycosphingolipids is attached by a glycosidic linkage to the terminal hydroxyl group of ceramide. In most animals, there are simple neutral glycosphingolipids with one or two sugar residues such as glucose (GIC) or galactose (Gal), intermediate molecular weight glycosphingolipids containing $\mathrm{N}$-acetylglucosamine (GlcNAC) or $\mathrm{N}$-acetyl-galactosamine (GaINAc) as well as glucose and galactose, some of which have terminal or branched fucose (Fuc) or N-acetylneuraminic acid (NeuAc) residues, and high molecular weight substances with 20 or more glycose residues. Glycosphingolipids with more than 6 sugar residues generally have a repeating unit that forms the skeleton of the carbohydrate chain and to which fucose and $\mathrm{N}$-acetylneuraminic acid as well as other sugars may be attached.

Simple arithmetic calculations indicate the enormous diversity of structures that are theoretically possible with a few different sugar consitutents, disregarding the heterogeneity of the ceramide moiety. For example, there could be about 500 million different glycosphingolipids, containing a core of 5 sugar residues (Glc, Gal, GICNAc and GaINAc combinations) in the pyranose ring form and one or two Fuc or NeuAc residues. The sugars do not appear to be chosen randomly by the biosynthetic enzymes, however, and some of the sugars are found exclusively in glycosphingolipids as a single ring size (pyranose) and anomeric configuration. Most glycosphingolipids of mammalian origin contain one of the core structures shown in Table II.

Complex fucoglycosphingolipids and sialoglycosphingolipids (gangliosides) are derived from these core structures and larger skeleton structures with several repeating disaccharide units of the lacto or neolacto type. Several recent reviews contain detailed information about the chemical structures of a large number of glycosphingolipids (5-7).

Some examples of recently characterized glycosphingolipids serve to illustrate the diversity of structures that occur in normal and transformed cells. Rat hepatoma cells and murine lymphoma cells contain a novel disialoganglioside (I) which has never been found in normal tissues $(8,9)$.

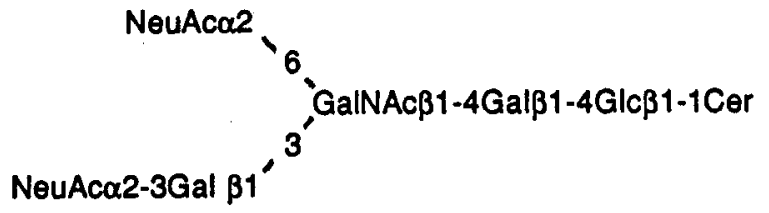

Human adenocarcinoma contains a somewhat similar disialoganglioside (II) but the core structure is of the lacto series rather than the ganolio series and it also contains a fucose residue (10). Metastatic cells in the liver of a colon cancer patient, on the other hand, contain a neutral fucoglycosphingolipid (III) of the neolacte type and a monsialoganglioside (IV) derived from III (11). Bovine buttermilk was found to contain a 


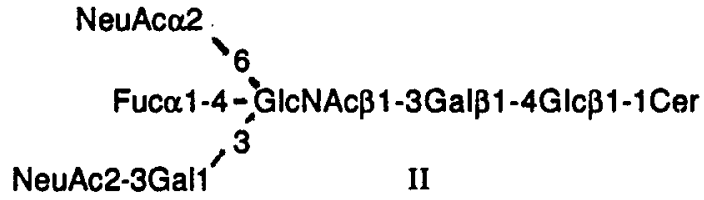

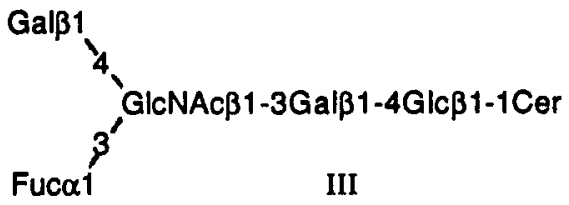

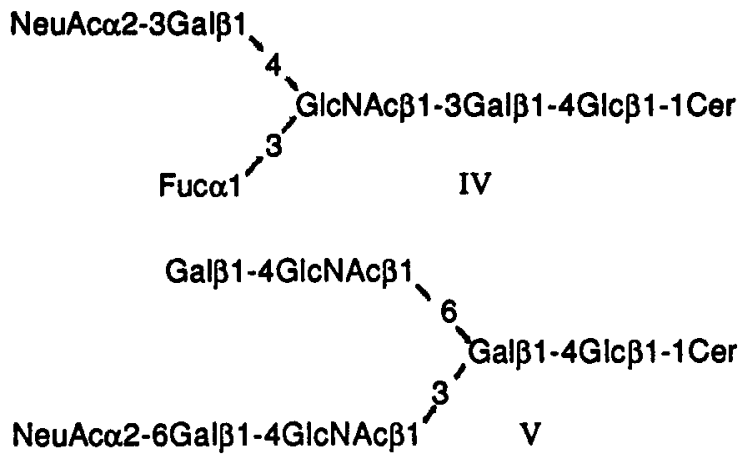

sialoglycosphingolipid (V) with a neolacto core structure and an unusual branch derived from a 1,6-linked Galß1-4GICNAC disaccharide residue (12). The same kind of branching pattern has been found to occur in larger glycosphingolipids with blood group-active substituents at the non-reducing end. One such structure (VI) was isolated recently from rabbit erythrocytes (13).

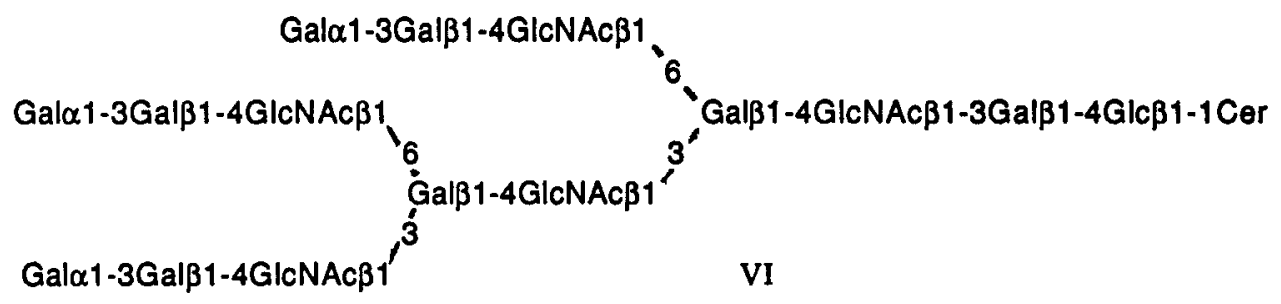

With improved methods of isolation and techniques for chemical structural analysis at the microgram level, the number of well-characterized glycosphingolipids has grown dramatically during the past decade. As of 1961, there were only 6 glycosphingolipids with definitive structures, even though perhaps another 10 or 15 compounds had been observed by chromatographic techniques. By 1975, the number had grown to 57, and as of July, 1988 approximately 215 glycosphingolipids have been completely characterized (14). Methods that have been used for structural analysis have included gas chromatographic analysis of methyl glycosides recovered after methanolysis (composition); GC-MS analysis of partially methylated alditol acetates after permethylation, aqueous hydrolysis, reduction and acetylation (positions of glycosidic linkages); sequential enzymatic hydrolysis with exoglycosidases (arrangement of sugars and anomeric configurations); proton NMR (anomeric configurations and other structural information from reporter signals); thin-layer chromatography with immuno- or lectin-staining (complete structures of certain ligands or epitopes); and direct mass spectral analysis of underivatized glycosphingolipids by a "soft ionization" technique such as liquid SIMS or fast-atom-bombardment (FAB), which give composition and sugar arrangement with as little as 1 microgram of a compound. The sensitivity of mass spectral analysis of glycosphingolipids with more than 10 sugar residues may be enhanced by analysis of permethylated or permethylated and $\mathrm{LiAlH}_{4}$ reduced samples $(15,16)$. The fragmentation pattern in the positive ionization mode of a blood group A-specific hexaglycosylceramide after permethylation and reduction would be expected to include a series of ions derived by random cleavage of the glycosidic linkages with charge retention on a ring oxygen atom and a companion series with loss of methanol (as shown in structure VII).

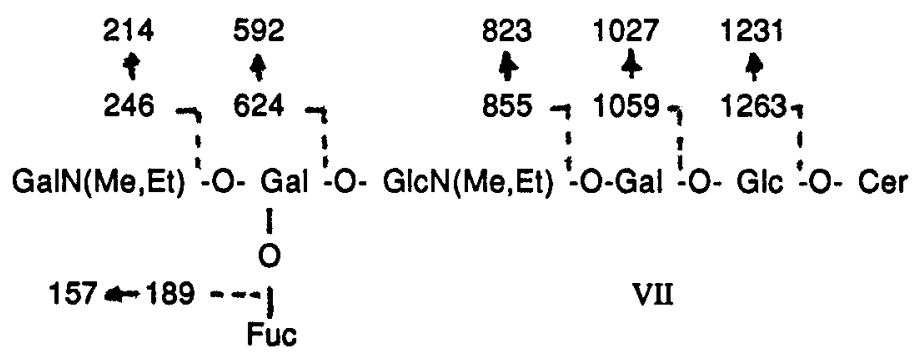


In the negative ion mode, the FAB mass spectrum of an underivatized ganglioside from murine lymphoma cells (VIII) contained a molecular ion $(\mathrm{M}-\mathrm{H})^{-}$at $\mathrm{m} / \mathrm{z} 1808$ for a ceramide, consisting of d18:1 (4-sphingenine) and 16:0 (palmitic acid), and a carbohydrate chain containing 3 hexose units, $1 \mathrm{~N}$-acetylhexosamine unit, and $2 \mathrm{~N}$-acetylneuraminic acid units

(17). The ceramide residue gave an ion at $\mathrm{m} / \mathrm{z} 536$ and there were a series of ions containing ceramide and partial carbohydrate chains at $\mathrm{m} / \mathrm{z} 698$ (Glc-Cer), 860 (Gal-Glc-Cer), 1355 (NeuAc-GalNAc-Gal-Glc-Cer), and 1517 (M-NeuAc). The absence of an ion at $\mathrm{m} / \mathrm{z} 1151$ (NeuAc-Gal-Glc-Cer) and the presence of a pair of ions at $\mathrm{m} / 2964$ and 962 distinguished this ganglioside from an isomeric disialoganglioside (Gd1a) with the structure NeuAc $\alpha 2-3 G a l \beta 1-3 G I N A c \beta 1-4$ (NeuAc $\alpha 2-3) \beta G a l \beta 1-4 G \mid c \beta 1-1$ Cer.

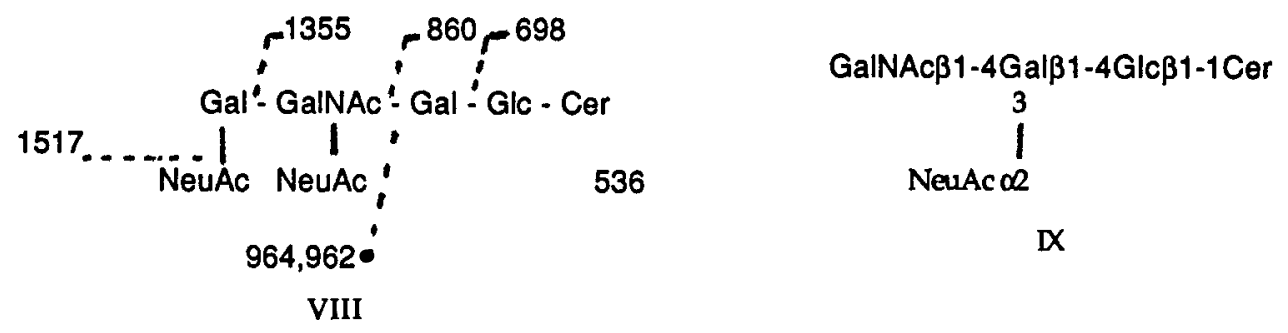

In some cases, the ceramide residue of glycosphingolipids might contain species of the same molecular weight but with different constituents, such as two molecular species of GM2 ganglioside from bovine brain (IX). The negative ion FAB spectrum (Fig. 2) of this ganglioside contained molecular ions $(\mathrm{M}-\mathrm{H})^{-}$at $\mathrm{m} / \mathrm{z} 1382$ for a species with ceramide containing 4-sphingenine (d18:1) and stearic acid (18:0) and at $\mathrm{m} / \mathrm{z} 1410$ for a species with a ceramide that could be either d18:1 and 20:0 or d20:1 and 18:0. Ohashi et al. (18) have reported that the positive $F A B$ spectra of glycosphingolipids contain a reporter ion $\left(\mathrm{Z}^{+}\right)$ at $\mathrm{m} / 2264$ or 292 for d18:1 or d20:1, respectively. This ion was also observed by Domon and Costello (19), who designated it the $W^{\mathrm{i}}$ ion. We have had difficulty finding this ion in the positve $F A B$ spectra of gangliosides, but it is a prominent daughter ion in the collision-assisted decomposition (CAD) of a parent ceramide ion in an MS/MS spectrum, as noted by Domon and Costello (19). In Fig. 2, the positive ion FAB CAD MS/MS spectrum of the ceramide ion $(\mathrm{m} / \mathrm{z} 576)$ of bovine brain $G M 2$ ganglioside shows $\mathrm{Z}^{+}\left(W^{\mathrm{ii}}\right)$ ions at $\mathrm{m} / \mathrm{z} 264$ and 292, indicating that the molecular species at $\mathrm{m} / \mathrm{z} 1410$ is, in fact, a mixture containing both d18:1-20:0 and d20:1-18:0, with the latter species being the predominant constituent. Such experiments, which can be carried out with as little as $0.5 \mathrm{ug}$ of material, overcome the difficulty of distinguishing sample ions from matrix ions and reveal structural details that could not otherwise be defined at this sensitivity.

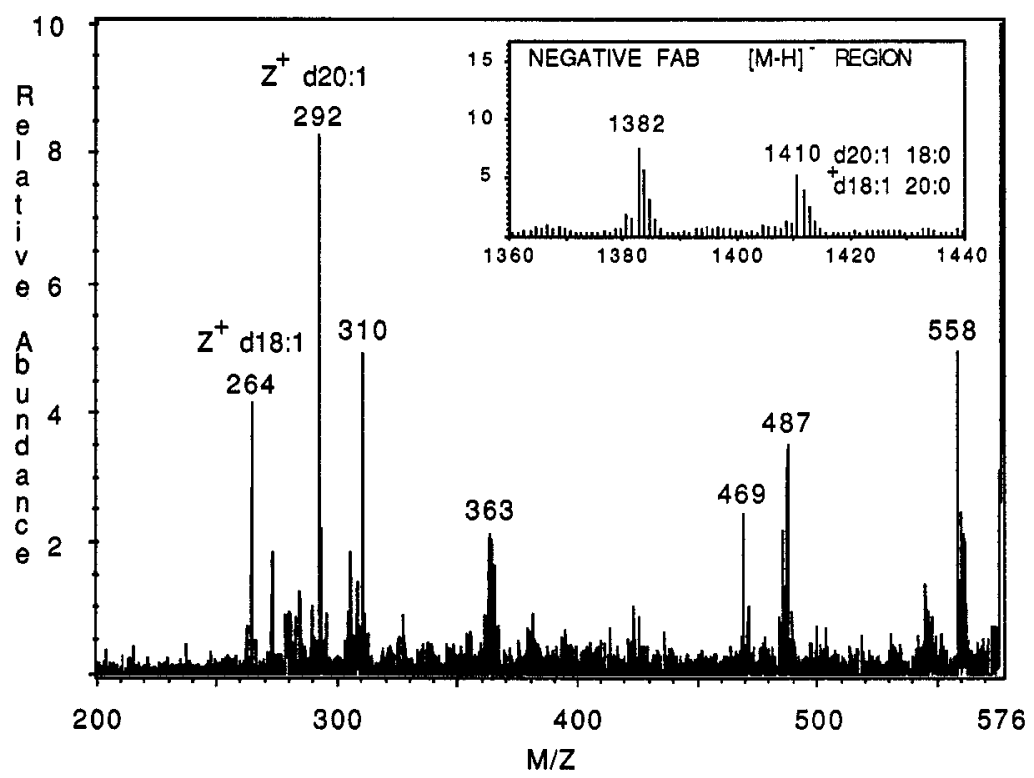

Fig, 2. Positive FAB CAD MS/MS of ceramide from bovine brain GM2 ganglioside. 


\section{FUNCTION}

The levels of glycosphingolipids on the extracellular aspect of the plasma membrane of cells are generally much lower than those of glycerophospholipids and cholesterol. Although it is not impossible that glycosphingolipids have a role in defining the overall fluidity and stability of the membrane, it seems more likely that they are involved in other phenomena at the cell surface, involving interactions where the three-dimensional structures of the carbohydrate chains are determinants of function. It is well-known that one of the polypeptide chains of cholera toxin, called the binding or B subunit, specifically interacts with GM1 ganglioside as an initial event in the activation of a membrane-bound adenyl cyclase by the active or $A$ subunit of the toxin (20). Similarly, other bacterial toxins and viruses appear to bind to specific carbohydrate structures that occur in various glycosphingolipids, and bacterial surface proteins have been shown to have exquisite specificity in the recognition and binding of bacteria to cell membrane glycosphingolipids (21). Other biologic activities of glycosphingolipids have been proposed, such as cell differentiation markers, modulation of cell proliferation, and immune recognition. As a particular focus of this review, the role of gangliosides in the regulation of growth of cultured cells will be considered.

In several lines of normal and transformed cells, exogeneously added gangliosides inhibit the mitogenic activity of growth factors (22). This effect appears to involve an extension of the quiescent or G1 phase of the cell cycle (23). The mechanism of ganglioside inhibition of growth is not completely understood, as yet, but experiments in vitro on isolated plasma membrane fractions suggest that gangliosides can negatively modulate the tyrosine kinase domain of growth factor receptors (24). This activity appears to be quite specific in the sense that GM1 ganglioside is most active with platelet-derived growth factor while GM3 ganglioside is most active with epidermal growth factor (22). It is possible that the function of other membrane-associated signal-transducing proteins are similarly modulated by specific glycosphingolipids, which would provide a partial explanation for the structural diversity that is found in mammalian cells. We have speculated that the modulating effect of such glycosphingolipids might be controlled by extracellular glycosidases that are 1) secreted by cells at certain densities of growth and/or phases of the cell cycle, 2) activated from a pro-enzyme form by extracellular proteases, and 3) modify the structures of the carbohydrate chains of specific glycosphingolipids and therefore their role in the modulation of receptors and other functional proteins of cell surface. To explore this possibility we have studied GM3 ganglioside metabolism in cultured human fibroblasts, which are growth-stimulated by epidermal growth factors (EGF). Some of our results have been reviewed (25).

If endogenous GM3, the principal ganglioside of human fibroblasts, has the same inhibitory effect on EGF receptor tyrosine phosphokinase activity as exogenous GM3, we reasoned that cells would be unable to progress through the cell cycle after EGF stimulation until the inhibitory effect of GM3 could be relieved in some manner. Although physical separation by lateral diffusion, shedding, or selective internalization of the GM3 are possible, metabolism seems to be a more likely explanation. To test this hypothesis, fibroblasts were pulse-labeled with radioactive precursors of the sialic acid and ceramide moieties, and the fate of the endogenously produced, doubly labeled GM3 was followed during a chase period of 24 hours (26). During this time there was a loss of substantial amounts of ${ }^{14} \mathrm{C}$ label from the sialic acid residue without any appreciable loss of ${ }^{3} \mathrm{H}$ label from the ceramide residue of GM3, and labeled free sialic acid accumulated in the medium. These results suggested the possibility that GM3 could be metabolized to lactosylceramide and sialic acid by an extracellular sialidase.

Sialidase activities with $\mathrm{GM} 3$ as the substrate were demonstrated in cell-conditioned medium and the plasma membrane fraction of the fibroblasts (26). At pH 4.5, the sialidase activities were 4.1 and $38 \mathrm{pmol}$ per hour per $\mathrm{ml}$ of medium at sparse and preconfluent densities, respectively. The $\mathrm{pH}$ versus activity profiles suggested the presence of another sialidase in the medium; this form had an optimal activity at pH 6.5 and was measureable only in actively growing cultures (26). Plasma membrane fractions contained about 10 times greater levels of the $\mathrm{pH} 4.5$ sialidase activity, which were not affected by cell density. It has not been possible as yet to determine which of these activities is responsible for GM3 turnover in these cells.

To test the hypothesis that GM3 metabolism may be important in the regulation of growth, we have tested the effect of a potent sialidase inhibitor, 2-deoxy-2,3-dehydro-N-acetylneuraminic acid (NeuAc2en), on cell growth (27). Treatment of fibroblasts with this compound resulted in the inhibition of the extracellular sialidase activity for up to 24 hours 
and marked inhibition of growth in a concentration-dependent manner. Moreover, the effect of the inhibitor was reversible. When serum-starved cells were incubated in fresh medium containing $80 \mu \mathrm{M}$ NeuAc2en, DNA synthesis was completely inhibited for 24 hours. Removal of the inhibitor resulted in S phase initiation with a peak of DNA synthesis 18 hours later, indicating that the cells were blocked in the G1 phase of the cell cycle by the sialidase inhibitor (27).

Other known sialidase inhibitors are being tested as growth inhibitors, and efforts are in progress to synthesize new inhibitors, some of which might react covalently at the active sites of sialidases and thus impart permanent inhibition.

Little is known as yet about the generality of this phenomenon. We have not been able to inhibit murine 3T3 cells with NeuAc2en, but the growth of a highly tumorigenic glioma cell line was inhibited (28). One must consider the possibility that such agents may have a clinical application in retarding the growth of malignant tumors in vive.

\begin{abstract}
Acknowledgements
I am grateful to M. Micke and D. Gage for helpful discussions and assistance in obtaining mass spectra, to $S$. Ladisch for the generous samples of gangliosides used in studies of FAB mass spectral behavior of gangliosides, and to my associates (S. Usuki, P. Hoops and S-C. Lyu) for their work with me on GM3 ganglioside metabolism and regulation of cell growth. This research was supported in part by research grants (DK 12434 and RR 00480) from the National Institutes of Health.
\end{abstract}

\title{
REFERENCES
}

1. H. E. Carter, F. J. Glick, W. P. Norris and G. E. Phillips, L_Biol Chem, 170, 285 (1947).

2. E. Klenk, Z. physiol. Chem, 235, 24 (1935).

3. R. Kuhn and H. Wiegandt, Chem. Ber., 96, 866 (1963).

4. Nomenclature was published in LLipid Res., 19, 121 (1977).

5. J. N. Kanfer and S. Hakomori, Sohingolipid Biochemistry (1983), Plenum Press, New York, pp 1-165.

6. H. Wiegandt, in Glycelipids (1982), ed. H. Wiegandt,

7. R. W. Ledeen and R. K. Yu, in Methods in Enzymology (1982), Vol. 83, ed. V. Ginsburg, Academic Press, New York, pp 139-191.

8. K. Murayama, S. B. Levery, V. Schirrmacher and S. Hakomori, Cancer Res., 46, 1395 (1986).

9. T. Taki, Y. Hirabayashi, H. Ishikawa, S. Ando, K. Kon, Y Tanaka and M. Matsumoto, L.Biol Chem., 261, 3075 (1986).

10.E. Nudelman, Y. Fukushi, S. B. Levery, T Higuchi and S. Hakomori, J. Biol.Chem, 261 5487 (1986).

11.T. Taki, M. Takamatsu, A. Myoga, K. Tanaka, S. Ando and M. Matsumoto, L.Biochem, 103 , 998 (1988).

12.K.Takamizawa, M. Iwamori, M. Mutai and Y. Nagai, J. Biol. Chem, 261, 5625 (1986).

13. H. Egge, M. Kordowicz, J. Peter-Katalinic and P. Hanfland, J.Biol Chem, 260, 4927 (1985).

14.B. Macher, private communication.

15.S. Hakomori, Chem. Phys. Lipids, 5, 96 (1970).

16.K-A. Karlsson, I. Pascher, W. Pimellot and B. E. Samuelsson, Biomed. Mass Spectrem., 1 , 49 (1974).

17.C. C. Sweeley, D. A. Gage and S. Ladisch, Abstracts Japanese Medical Mass Spectrometry Symp., Sept. 27-30, 1988.

18.Y. Ohashi, M. Iwamori, T. Ogawa and Y. Nagai, Biochemistry 26, 3990 (1987).

19.B. Domon and C. E. Costello, Biochemistry, 271534 (1988).

20.C. A. King and W. E. van Heyningen, J.Inlect. Dis., 12Z, 639 (1973).

21.K. Bock, M. E. breimer, A. Brignole, G. C. Hansson, K-A. Karisson, G. Larson, H. Leffler, B. E. Samuelsson, N. Stromberg, C. Svanborg-Eden and J. Thurin, J.Biol Chem, 260, 8545 (1985).

22.E. G. Bremer, S. Hakomori, D. F. Bowen-Pope, E. Raines and R. Ross, L Biol Chem, 259, 6818 (1984).

23.T. Dhsawa and T. Senshu, Exptl_Cell Res., 173, 49 (1987).

24.E. G. Bremer, J. Schlessinger and S. Hakomori, J. Biol.Chem. 261, 2434 (1986).

25.S. Usuki and C. C. Sweeley, Indian J. Biochem. Biophrs., 25, 102 (1988).

26.S. Usuki, S-C. Lyu and C. C. Sweeley, L.Biol. Chem, 263, 6847 (1988).

27.S. Usuki, P. Hoops and C. C. Sweeley, J_Biol. Chem., 263, 10595 (1988).

28.J. Moskal, private communication. 